\title{
VLT/NACO adaptive optics imaging of GSS 30 IRS1: a protostellar binary system? ${ }^{\star}$
}

\author{
X. P. Chen, R. Launhardt, and Th. Henning
}

\begin{abstract}
Max Planck Institute for Astronomy, Königstuhl 17, 69117 Heidelberg, Germany
e-mail: [chen;rlau; henning] @mpia-hd.mpg.de
\end{abstract}

Received 15 December 2006 / Accepted 28 August 2007

\section{ABSTRACT}

\begin{abstract}
Aims. We present high-resolution near-infrared images of the low-mass Class I young stellar object GSS 30 IRS1. Methods. The data were retrieved from the ESO archive and acquired in the $H K_{\mathrm{s}} L^{\prime}$-bands with the adaptive optics system NACO at the ESO Very Large Telescope.

Results. We discover two separate components in GSS 30 IRS1. The angular separation between the two components is $\sim 0$ '. 15 , corresponding to a projected linear separation of $24 \mathrm{AU}$ at a distance of $160 \mathrm{pc}$. The brightness contrasts between the two components are $\sim 1.4 \mathrm{mag}$ at $H$ band, $1.5 \mathrm{mag}$ at $K_{\mathrm{s}}$ band, and $2.1 \mathrm{mag}$ at $L^{\prime}$ band. The two components detected in GSS 30 IRS1 could represent two scattered light structures related to an outflow-disk complex or two young stellar objects embedded in a common disk. Here we discuss these two possibilities, as well as the CO ro-vibrational emission from GSS 30 IRS1.
\end{abstract}

Key words. binaries: close - stars: formation - stars: individual: GSS 30 IRS1

\section{Introduction}

GSS 30 IRS1 (hereafter also IRS1) is one of the best-studied young stellar objects (YSOs) in the $\rho$ Ophiuchus molecular cloud core at a distance of $\sim 160 \mathrm{pc}$. It has been classified as a low-mass Class I source based on its spectral energy distribution (Grasdalen et al. 1973; Elias 1978; Wilking et al. 1989) and bolometric luminosity $\left(L_{\mathrm{bol}}=21 L_{\odot}\right.$; Bontemps et al. 2001). Near-infrared (NIR) polarimetric studies have shown that IRS1 is surrounded by a large ( $2000 \mathrm{AU})$ disk-like envelope and a smaller $(\sim 150 \mathrm{AU})$ circumstellar disk, which is inclined $\sim 25^{\circ}$ away from the plane of the sky (Castelaz et al. 1985; Tamura et al. 1991; Weintraub et al. 1993; Chrysostomou et al. 1996). Strong $1.3 \mathrm{~mm}$ dust continuum emission was detected from IRS 1 with an integrated flux density of $\sim 100 \mathrm{mJy}$, indicative of a circumstellar mass of $\sim 0.03 M_{\odot}$ (André \& Montmerle 1994). Zhang et al. (1997) detected a flattened molecular core in $\mathrm{C}^{18} \mathrm{O}$ and ${ }^{13} \mathrm{CO}(J=1-0)$, in roughly the same orientation as the circumstellar disk. Recently, Pontoppidan et al. (2002) detected bright CO ro-vibrational emission from IRS1, suggesting a strong accretion shock in the inner 10-50 AU of the circumstellar disk. Tamura et al. (1990) reported high velocity CO emission at $115 \mathrm{GHz}$ around IRS 1 in a region of roughly $5^{\prime}$ in extent. However, since both the blue- and red-shifted molecular gas are located to the south of GSS 30, the origin of the molecular outflow could not be identified. Here we present new NIR observations of GSS 30 IRS1, conducted with NACO at the ESO Very Large Telescope (VLT). Based on the high-resolution images, we investigate the inner structure of this young stellar object.

* Based on data from the ESO/ST-ECF Science Archive Facility.

\section{Observations and data reduction}

The data were obtained from the ESO/ST-ECF Science Archive Facility ${ }^{1}$. GSS 30 IRS1 [RA $=16^{\mathrm{h}} 26^{\mathrm{m}} 21.5^{\mathrm{s}}$, Dec $=$ $-24^{\circ} 23^{\prime} 07^{\prime \prime}$ (J2000)] was observed with NACO (Lenzen et al. 2003; Rousset et al. 2002) at the ESO VLT (UT4) on Cerro Paranal in Chile on March 21st and June 1st 2003. The detector of NACO is an Aladdin InSb $1024 \times 1024$ pixel array. The cameras used were S13, S27, and L27, which provide pixel scales of $13.25,27.03$, and 27.12 mas pixel ${ }^{-1}$, respectively. The fields of view of the S13, S27, and L27 cameras are $\sim 14^{\prime \prime} \times 14^{\prime \prime}$, $28^{\prime \prime} \times 28^{\prime \prime}$, and $28^{\prime \prime} \times 28^{\prime \prime}$, respectively.

The observations were carried out in $H$-band $(1.66 \mu \mathrm{m})$, $K_{\mathrm{s}}$-band $(2.18 \mu \mathrm{m})$, and $L^{\prime}$-band $(3.80 \mu \mathrm{m})$. Standard stars SAO 245532 and HD 161742, observed on June 1st 2003, were used for photometric calibration. Observation parameters are summarized in Table 1. The data reduction was carried out using self-developed IDL scripts. After bad-pixel filtering, flat-field correction, and sky removal, the individual dithering positions were combined into a resulting mosaic image. Further analysis and figures were done with the GILDAS ${ }^{2}$ software package.

\section{Results}

Figure 1a shows the VLT/NACO S27/H-band image of IRS1. A bipolar nebulosity is seen around IRS1, with a bright doublewing lobe extending to the northeast and a fainter lobe extending to the southwest. Figure $1 \mathrm{~b}$ shows a higher resolution $\mathrm{S} 13 / K_{\mathrm{s}}$-band image of the central part around IRS1. In this image IRS1 is resolved into two objects. In the following, we refer to the brighter eastern object as IRS1 A and to the western object as IRS1 B. Figure 1c shows the L27/L'-band image of IRS1.

\footnotetext{
1 http://archive.eso.org

2 http://www.iram. fr/IRAMFR/GILDAS
} 
Table 1. NACO observation logs of GSS 30 IRS1 and photometric calibration stars SAO 245532, HD 161743.

\begin{tabular}{lcccccc}
\hline \hline Source & $\begin{array}{c}\text { Obs. date } \\
\text { (UT Date) }\end{array}$ & $\begin{array}{c}\text { NACO } \\
\text { Camera }\end{array}$ & $\begin{array}{c}\text { Obs. } \\
\text { Band }\end{array}$ & $\begin{array}{c}D I T \times N^{1} \\
(\mathrm{~s})\end{array}$ & $\begin{array}{c}\text { Seeing } \\
\left({ }^{\prime \prime}\right)\end{array}$ & Airmass \\
\hline IRS1 & 2003 March 03 & $\mathrm{S} 13$ & $K_{\mathrm{s}}$ & $60.0 \times 4$ & 0.81 & 1.01 \\
\hline IRS1 & 2003 June 01 & $\mathrm{S} 27$ & $H$ & $80.0 \times 4$ & 0.67 & 1.08 \\
$\mathrm{IRS1}$ & 2003 June 01 & $\mathrm{S} 27$ & $K_{\mathrm{s}}$ & $10.0 \times 4$ & 0.75 & 1.12 \\
$\mathrm{IRS1}$ & 2003 June 01 & $\mathrm{L} 27$ & $L^{\prime}$ & $0.175 \times 18$ & 1.00 & 1.03 \\
$\mathrm{SAO} 245532$ & 2003 June 01 & $\mathrm{S} 27$ & $K_{\mathrm{s}}$ & $0.5 \times 5$ & 1.06 & 1.34 \\
$\mathrm{SAO} 245532$ & 2003 June 01 & $\mathrm{S} 27$ & $H$ & $0.5 \times 5$ & 0.92 & 1.33 \\
$\mathrm{HD} 161743$ & 2003 June 01 & $\mathrm{L} 27$ & $L^{\prime}$ & $0.18 \times 5$ & 1.18 & 1.04 \\
\hline
\end{tabular}

Note 1. DIT and $N$ correspond to the exposure integration time and the number of exposures, respectively.
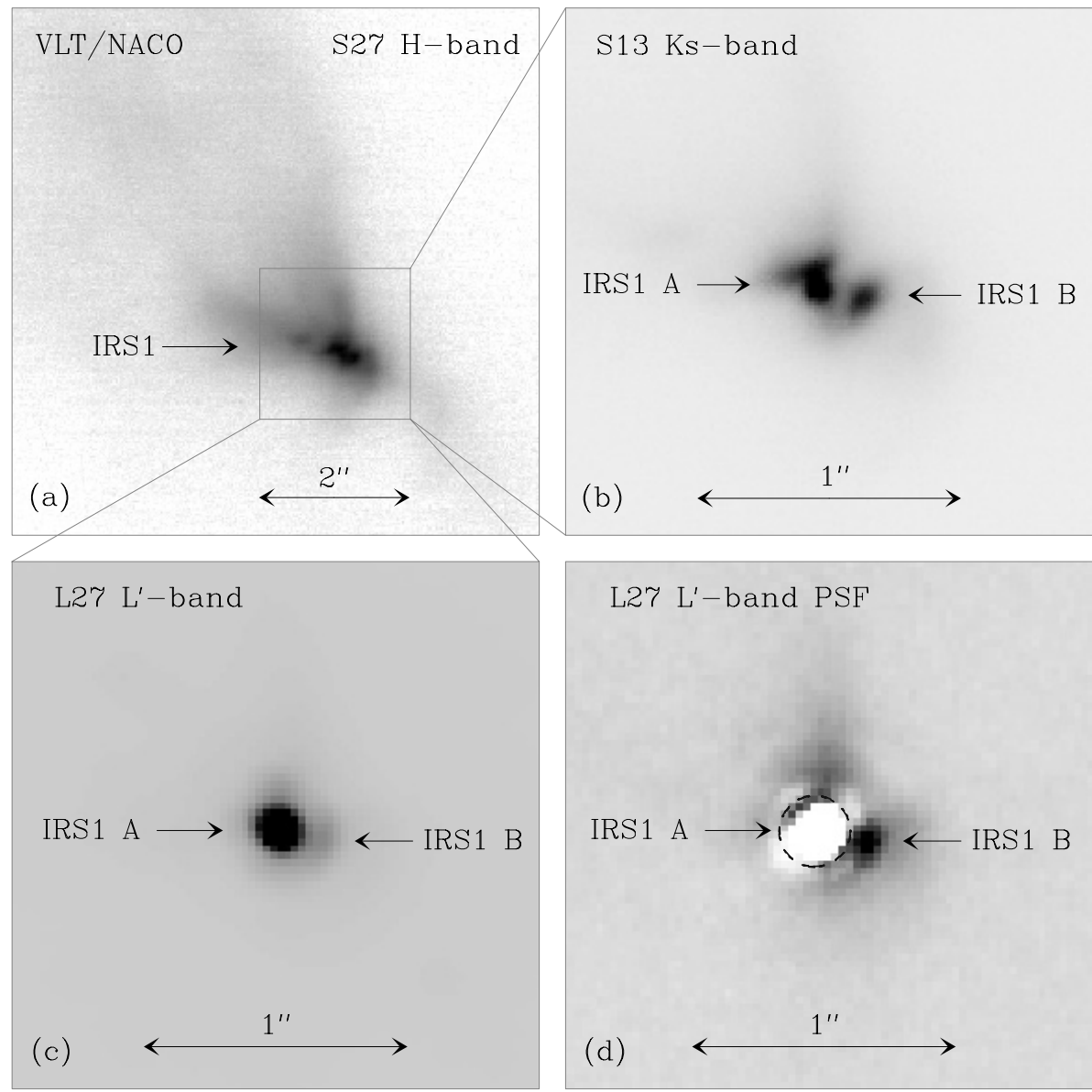

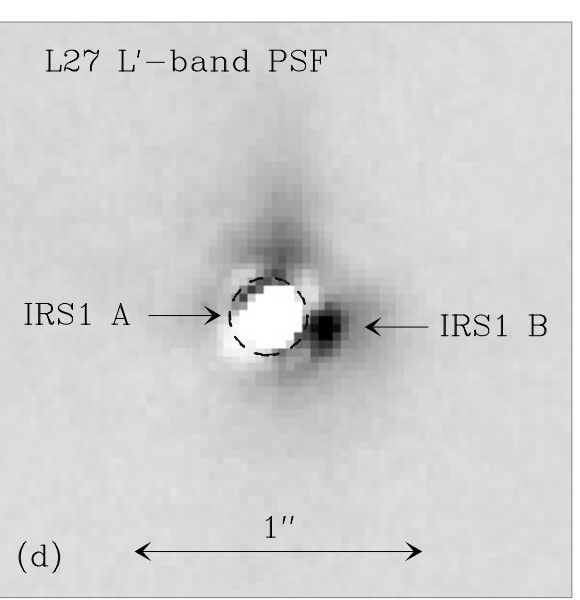

Fig. 1. a) VLT/NACO S27 image of GSS 30 IRS1 in the $H$-band $(1.65 \mu \mathrm{m})$. b) S13 $K_{\mathrm{s}}$-band $(2.18 \mu \mathrm{m})$ image of the inner region around IRS1. The positions of IRS1 A and IRS1 B are indicated with letters A and B, respectively. c) L27 $L^{\prime}$-band $(3.80 \mu \mathrm{m})$ image of IRS1. d) L27 $L^{\prime}$-band PSF-subtracted image of IRS1. For all the images, north is up and east is to the left. The scales are marked in each map.
In this image a strong point-like object is seen at the position of IRS1 A, but fainter emission is also detected at the position of IRS1 B. To enhance the contrast on the faint source, we subtracted the PSF from IRS1 A. In this $L^{\prime}$-band PSF-subtracted image, a point-like object becomes visible at the position of IRS1 B (see Fig. 1d).

To view the structure in detail, in Fig. 2 we show the contour maps of the inner region around IRS1. At all $H K_{\mathrm{S}} L^{\prime}$ bands, IRS1 is resolved into two objects. However, both IRS1 A and IRS1 B show extended structures at $H K_{\mathrm{s}}$ bands (see Figs. $2 \mathrm{a}$ and $2 \mathrm{~b}$ ). Figure 2c shows the contour of the $L^{\prime}$-band PSF-subtracted image, overlaid with the contours from Figs. $1 \mathrm{~b}$ and $1 \mathrm{c}$. At $L^{\prime}$ band, both objects show point-like structures. Furthermore, we find that IRS1 A and IRS1 B detected at $K_{\mathrm{S}}$ band are spatially coincident with each $L^{\prime}$ band source. Nevertheless, there is a small offset ( $\sim 10$ mas) between the peaks of IRS1 B in the $S 13 / K_{\mathrm{s}}$-band image and L27/L'-band image (see Fig. 2c), which could be due to the differences in resolution and wavelength between the cameras S13 and L27.

The photometry of IRS 1 and calibration stars was performed with the GILDAS package. For comparison with earlier papers, we first treat IRS1 as a single source and integrate the flux densities enclosed by the FWHM levels. The integrated flux densities are then converted into magnitudes by applying the same method to the standard stars (see Table 2). The derived magnitudes of "IRS1" compare well with the results in Weintraub et al. (1993) and Barsony et al. (1997). In order to obtain the magnitudes of IRS1 A and IRS1 B, a number of assumptions have to be made because of their extended structures and strong scattering in nearby nebulosity. At $H K_{\mathrm{s}}$ bands, we integrate the flux densities enclosed by the $80 \%$ level ( $H$ band $)$ and $70 \%$ level $\left(K_{\mathrm{s}}\right.$ band) of the peak intensities (where the two objects are separated; see Fig. 2) and then estimate flux ratios between the two objects. By combining "IRS1" magnitudes and the flux ratios, we derive individual magnitudes of IRS1 A and IRS B. At $L^{\prime}$ band, the 

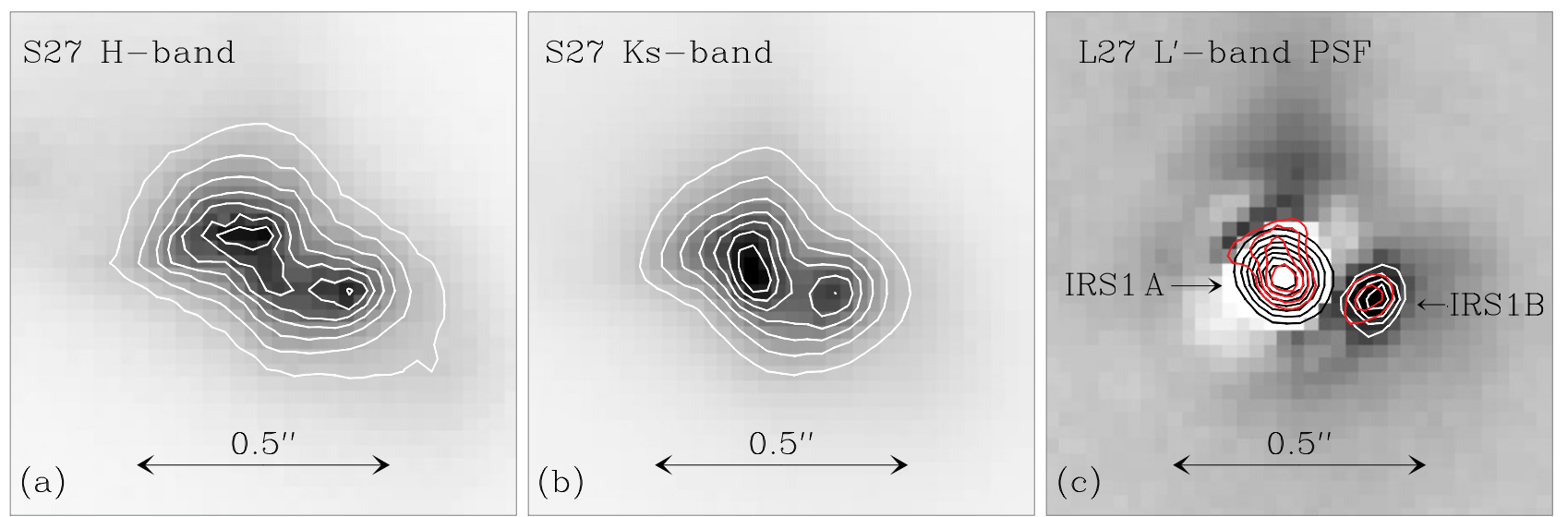

Fig. 2. a) $\mathrm{S} 27 / H$-band contour image. Contours start from $30 \%$ to $90 \%$, in steps of $10 \%$ of the peak intensity. b) Same as Fig. 2 a, but at $S 27 / K_{\mathrm{s}}-$ band. c) $L^{\prime}$-band PSF-subtracted image. Contours (white) are at $60 \%, 70 \%, 80 \%$, and $90 \%$ of the peak intensity of IRS1 B. The image is overlaid with L27/L'-band contours (black; from $30 \%$ to $90 \%$, in steps of $10 \%$ of the peak intensity of IRS1 A) and S13/ $K_{\mathrm{s}}$-band contours (red; from $60 \%$ to $90 \%$, in steps of $10 \%$ of the peak intensity of IRS1 A).

Table 2. $H K_{\mathrm{s}} L^{\prime}$ magnitudes and colors of IRS1 A and IRS1 B. Data are from the VLT/NACO S27 camera.

\begin{tabular}{lccccc}
\hline \hline Source & $H$ & $K_{\mathrm{s}}$ & $L^{\prime}$ & $H-K_{\mathrm{S}}$ & $K_{\mathrm{s}}-L^{\prime}$ \\
\hline IRS1 & $10.9 \pm 0.1$ & $8.4 \pm 0.1$ & $7.84 \pm 0.02$ & $2.5 \pm 0.2$ & $0.6 \pm 0.1$ \\
IRS1 A & $11.3 \pm 0.2$ & $8.7 \pm 0.1$ & $7.98 \pm 0.02$ & $2.6 \pm 0.3$ & $0.7 \pm 0.1$ \\
IRS1 B & $12.7 \pm 0.3$ & $10.2 \pm 0.2$ & $10.1 \pm 0.03$ & $2.5 \pm 0.5$ & $0.1 \pm 0.2$ \\
\hline
\end{tabular}

magnitude of IRS1 B was calculated from the integrated flux density enclosed by the FWHM level in the PSF-subtracted image. The derived $H K_{\mathrm{s}} L^{\prime}$ magnitudes, uncertainties, and colors of IRS1 A and IRS1 B, are listed in Table 2. The brightness contrasts between IRS1 A and IRS1 B are 1.4 mag at $H$ band, $1.5 \mathrm{mag}$ at $K_{\mathrm{s}}$ bands $^{3}$, and $2.1 \mathrm{mag}$ at $L^{\prime}$ band, indicating that IRS1 A is "redder" than IRS1 B. However, it must be noted that large uncertainties exist in this photometry and the results can be referred only for qualitative analysis.

\section{Discussion}

\subsection{Two scattered light peaks?}

In general, Class I young stellar objects are still embedded in their remnant envelope, which produces large scattered light nebulosity and makes the interpretation of the NIR maps very complicated. In the case of GSS 30 IRS1, the northeast doublewing lobe has long been suggested as scattered light from the wall of a cavity evacuated by the molecular outflow, while the fainter southwest lobe is proposed to be obscured by a tilted circumstellar disk (Castelaz et al. 1985; Tamura et al. 1991; Weintraub et al. 1993; Chrysostomou et al. 1996).

Taking into account this outflow-disk complex, the two objects detected in IRS1 may represent two scattered light peaks from two dust clumps located on the outer wall of the outflow cavity. These two dust clumps could be separated by an optically thick ridge of dense material in the disk. This is a wellknown effect from optically thick circumstellar disks observed close to edge-on (see e.g., Wolf et al. 2003). Although a simple disk structure seems unlikely to account for the observations of GSS 30 IRS1, a similar behavior related to a partially optically thick nebula could not be ruled out here.

\footnotetext{
${ }^{3}$ In the deeper image observed by S13 camera, the brightness contrast is $\sim 1.0$ mag at $K_{\mathrm{s}}$ band.
}

\subsection{A protostellar binary system?}

Another possibility is that IRS1 A and IRS1 B represent two YSOs and compose a binary system embedded in a common disk. Indeed, the good agreement between the $S 13 / K_{\mathrm{s}}$-band image and L27/L'-band image (see Fig. 2c) suggests that the two objects represent actual stars rather than scattered light structures.

However, in contrast to the point-like structures seen at $L^{\prime}$ band, both objects show extended structures at $H K_{\mathrm{s}}$ bands (especially at $H$ band), which makes us still doubt their stellar qualifications. Unfortunately, the three-band NIR observations acquired so far are not sufficient to determine the natures of the two objects. Since the $1.3 \mathrm{~mm}$ continuum observations suggest a great deal of circumstellar dust around IRS1 (André \& Montmerle 1994), we speculate that this extended structure is due to the dust scattering in a small cocoon/halo (or even circumstellar disk) surrounding each component, the tidal truncation causing it to be non-spherical (see Lubow \& Artymowicz 2000; hereafter LA2000). At $L^{\prime}$ band, the dust opacity (scattering) is greatly reduced; here, the point-like structure of two objects is revealed.

Assuming IRS1 is a binary system, the angular separation and position angle (with respect to IRS1 A) were measured to be $154.4 \pm 5$ mas and $256^{\circ} .5 \pm 0.5$ in the $S 13 / K_{\mathrm{s}}$-band image, respectively. The angular separation corresponds to a linear projected separation of $\sim 24 \mathrm{AU}$ at a distance of $160 \mathrm{pc}$. Due to this small separation, IRS1 A and IRS1 B remained unresolved in previous binary surveys of the $\rho$ Ophiuchi molecular cloud (see Haisch et al. 2002; Duchêne et al. 2004).

\subsection{Where is the CO emission from?}

The bright $\mathrm{CO}$ ro-vibrational emission detected by Pontoppidan et al. (2002) suggests a strong accretion shock in the inner $10-50 \mathrm{AU}$ of IRS1. If the two objects detected here are 
scattered light structures from the dust clumps, it is likely that they are associated with this accretion shock and related to the $\mathrm{CO}$-emitting region (since the projected separation between IRS1 A and IRS B is in the right range).

On the other hand, if IRS1 is a binary system, according to the theoretical simulation in Artymowicz \& Lubow (1994), the inner radius of the circumbinary disk and the outer radii of the circumstellar disks would be 50-70 AU and 5-10 AU, respectively (taking the projected separation $24 \mathrm{AU}$ as the semimajor axis of the binary system). Thus, the accreting region suggested by Pontoppidan et al. is located exactly in the gap opened by the binary system, which seems to exclude the binary scenario. However, numerical simulations also show that the gas from the circumbinary disk could cross disk gaps in the form of accretion streams, feeding matter onto the binary system (see Artymowicz \& Lubow 1996 and LA2000). At the same time, there is a long standing argument in the observations that T Tauri stars with close stellar companions will show $\mathrm{CO}$ fundamental emission (NIR excess) from residual material within a dynamically cleared disk gap (see Carr et al. 2001; Mathieu et al. 2000; Najita et al. 2000, 2003). GSS 30 IRS1 is a young Class I YSO, which means much active accreting and more material survived from dynamical clearing. The $\mathrm{CO}$ emission detected in IRS1 could have originated from this accretion stream across the gap. This assumption does not only agree with the accretion shock suggested by the $\mathrm{CO}$ emission line, but also explains simultaneously why this accretion shock is variable, as suggested by Pontoppidan et al. (2002) and the variable water maser within 0.'3 ( 48 AU) of IRS1 (Claussen et al. 1996).

\section{Conclusions}

With high-resolution VLT/NACO observations, we discover two separate components in the Class I young stellar object GSS 30 IRS1. The angular separation between the two components is $\sim 0{ }^{\prime} 15$, corresponding to a projected linear separation of $24 \mathrm{AU}$ at a distance of $160 \mathrm{pc}$. The brightness contrasts between the two components are $\sim 1.4 \mathrm{mag}$ at $H$ band, $1.5 \mathrm{mag}$ at $K_{\mathrm{s}}$ band, and $2.1 \mathrm{mag}$ at $L^{\prime}$ band. The two components could represent two scattered light structures related to an outflow-disk complex or two young stellar objects embedded in a common disk. Both interpretations are equally convincing. More observations, e.g., high-resolution spectroscopy and longer-wavelength imaging, are needed in future to determine the natures of these two new components.

Acknowledgements. We thank the anonymous referee for many insightful comments and suggestions. This research is based on observations made with the European Southern Observatory telescopes obtained from the ESO/ST-ECF Science Archive Facility.

\section{References}

André, P., \& Montmerle, T. 1994, ApJ, 420, 837

Artymowicz, P., \& Lubow, S. H. 1994, ApJ, 421, 651

Artymowicz, P., \& Lubow, S. H. 1996, ApJ, 467, L77

Barsony, M., Kenyon, S. J., Lada, E. A., \& Teuben, P. J. 1997, ApJS, 112, 109

Bontemps, S., André, P., Kaas, A. A., et al. 2001, A\&A, 372, 173

Carr, J. S., Mathieu, R. D., \& Najita, J. R. 2001, ApJ, 551, 454

Castelaz, M. W., Hackwell, J. A., Grasdalen, G. L., et al. 1985, ApJ, 290, 261

Claussen, M. J. Wilking, B. A., Benson, P. J., et al. 1996, ApJS, 106, 111

Chrysostomou, A., Clark, S. G., Hough, J. H., et al. 1996, MNRAS, 278, 449

Duchêne, G., Bouvier, J., Bontemps, S., et al. 2004, A\&A, 427, 651

Elias, J. H. 1978, ApJ, 224, 453

Grasdalen, G. L., Strom, K. M., \& Strom, S. E. 1973, ApJ, 184, L53

Haisch, K. E., Barsony, M., Greene, T. P., \& Ressler, M. E. 2002, AJ, 124, 2841

Lenzen, R., Hartung, M., Brandner, W., et al. 2003, SPIE, 4841, 944

Lubow, S. H., \& Artymowicz, P. 2000, in Protostars and Planets IV, ed. V. Mannings, A. P. Boss, \& S. R. Russell (Tucson: Univ. Arizona Press), 731

Mathieu, R. D., Ghez, A. M., Jensen, E. L. N., \& Simon, M. 2000, in Protostars and Planets IV, ed. V. Mannings, A. P. Boss, \& S. R. Russell (Tucson: Univ. Arizona Press), 703

Najita, J. R., Edwards, S., Basri, G., \& Carr, J. 2000, in Protostars and Planets IV, ed. V. Mannings, A. P. Boss, \& S. R. Russell (Tucson: Univ. Arizona Press), 457

Najita, J. R., Carr, J. S., \& Mathieu, R. D. 2003, ApJ, 589, 931

Pontoppidan, K. M., Schöier, F. L., van Dishoeck, E. F., \& Dartois, E. 2002, A\&A, 393, 585

Rousset, G., Lacombe, F., Puget, P., et al. 2003, SPIE, 4839, 140

Tamura, M., Sato, S., Suzuki, H., et al. 1990, ApJ, 350, 728

Tamura, M., Gatley, I., Joyce, R. R., et al. 1991, ApJ, 378, 611

Weintraub, D. A., Kastner, J. H., Griffith, L. L., \& Campins, H. 1993, AJ, 105, 271

Wilking, B. A., Lada, C. J., \& Young, E. T. 1989, ApJ, 340, 823

Wolf, S., Padgett, D. L., \& Stapelfeldt, K. R. 2003, ApJ, 588, 373

Zhang, Q., Wootten, A., \& Ho, P. T. P. 1997, ApJ, 475, 713 